\title{
Esfingídeos (Lepidoptera, Sphingidae) do município de Beruri, Amazonas, Brasil
}

\author{
Catarina da Silva MOTTA ${ }^{1}$; Francisco Felipe XAVIER-FILHO ${ }^{1}$
}

\section{RESUMO}

Mariposas esfingídeas foram coletadas no Município de Beruri, região do baixo rio Purus, Estado do Amazonas, em três períodos 30/09-10/10/2002, 25-30/07/2003 e 29/11-08/12/2003. Foi utilizada uma lâmpada de luz mista de mercúrio de $250 \mathrm{~W}$, sobre um lençol branco, em noites de coletas de 12 horas consecutivas. Foram coletados 295 exemplares, identificados em 46 espécies de 20 gêneros. A tribo Dilophonotini foi a mais representada (23 espécies), seguida por Sphingini ( 9 spp.), Macroglossini (6 spp.), Philampelini (4 spp.), Ambulycini (3 spp.) e Acherontiini (1 sp.). Todas as espécies foram registradas pela primeira vez para o Município de Beruri. São registrados os esfingídeos que são pragas e aqueles considerados pragas em potencial.

PALAVRAS-CHAVE

Bombycoidea, Sphingidae, baixo rio Purus, Município de Beruri, Amazônia central.

\section{Hawkmoths (Lepidoptera, Spbingidae) from the District of Beruri, State of Amazonas, Brazil}

\begin{abstract}
Hawkmoths were collected at District of Beruri, low river Purus, State of Amazonas in threeperiods September-October/2002,July/ 2003 and November-December/2003. A250W mixed mercury vapor lamp was used to atract insects on a white sheet in the nights collecting periods of 12 consecutive hours. 295 specimens were collected of 46 species, and 20 genera were identified, with predominance ofDilophonotini (23 species) followed by Sphingini (9spp.), Macroglossini (6spp.), Philampelini (4spp.), Ambulycini (3 spp.) and Acheronthiini (1 sp.). These are the first records of sphingids for this locality. Species considered simple and/or potential pests were registered.
\end{abstract}

KEYWORDS

Bombycoidea, Sphingidae, low river Purus, District of Beruri, Central Amazon

${ }^{1}$ Coordenação de Pesquisas em Entomologia (CPEN), Instituto Nacional de Pesquisas da Amazônia (INPA), c.p. 478, 69011-970 Manaus, AM, Brasil e-mail:motta@inpa.gov.br 


\section{INTRODUÇÃO}

Os esfingídeos, comumente denominadas de fifes ou bruxas, como outros lepidópteros têm importante papel na dispersão de pólen de várias plantas, devido à nectarivoria dos adultos. Suas lagartas são herbívoras, desfolhadoras de espécies vegetais utilizadas pelo homem. Por seus hábitos alimentares, através da interação inseto-planta, são potenciais indicadores biológicos do estado de saúde de comunidades vegetais naturais (Motta, 1993).

\section{MATERIAL E MÉTODOS}

Três coletas foram realizadas em Beruri, região do baixo rio Purus e têm sua importância por serem os primeiros exemplares, dessas mariposas, coletados nesse município do

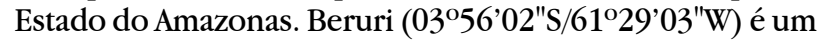
pequeno município localizado à margem direita do rio Purus (Fig. 1). Nessa área $\left(17.250 \mathrm{~km}^{2}\right)$ em que é predominante a floresta primária, possui na área urbana, uma população constituída principalmente pelos habitantes da sede que se

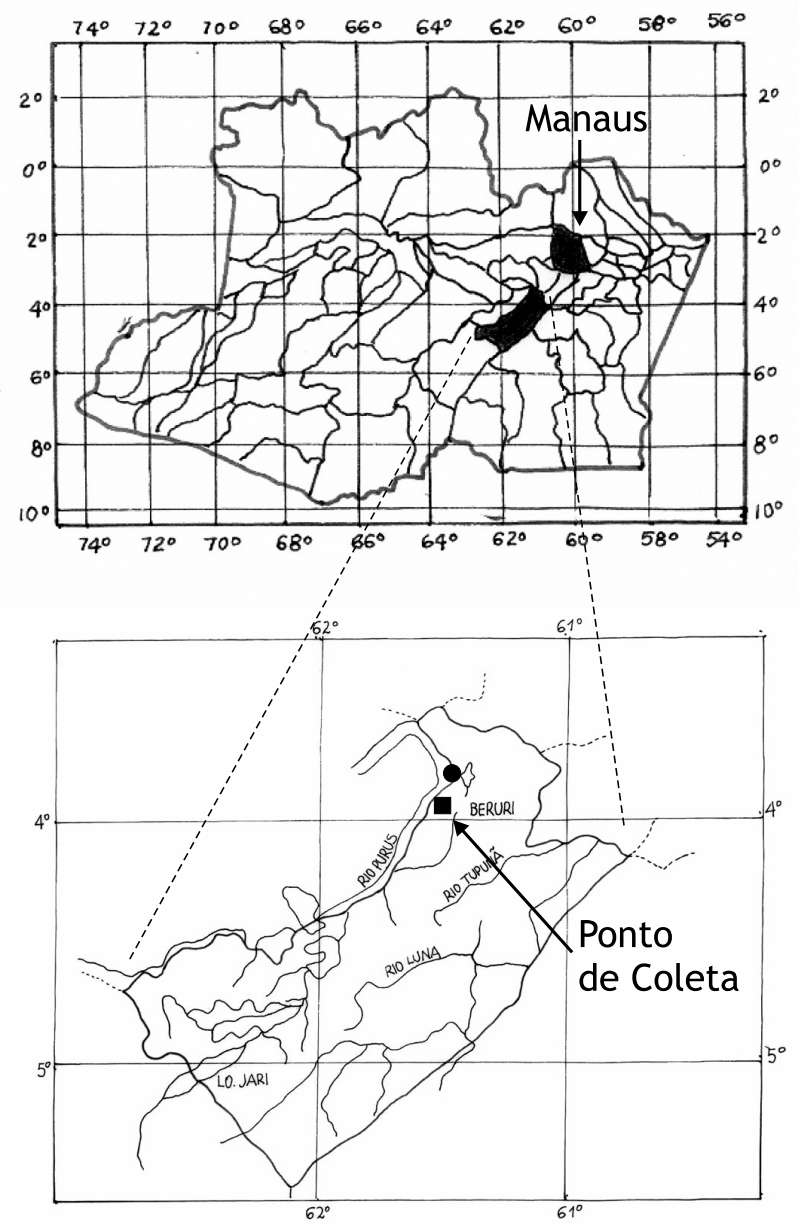

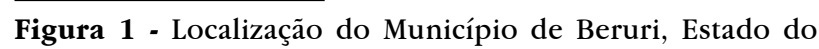
Amazonas, Brasil. somando aos ribeirinhos chega a 11.038 habitantes (IBGE 2000).

As coletas foram realizadas na Estrada do INCRA (Instituto Nacional de Colonização e Reforma Agrária), km 3, (0358'25'S/ 61025'02'W) em um sítio onde existem plantações de cupuaçuzeiros - Theobroma angustifolium (Wild. ex Spreng.) (Sterculiaceae) e cajueiros-Anacardium occidentalis Linnaeus (Anacardiaceae); um lençol branco de 1,40 x 1,40 m foi aberto em uma armação apropriada de alumínio e sobre este, como fonte atrativa, foi colocada uma luz mista de mercúrio de 250 W, em posição vertical. Este método já foi utilizado em levantamentos anteriores desses lepidópteros, sendo o mais indicado para a captura dos esfingídeos (Motta et al., 1991).

As coletas foram realizadas em três períodos 30/09-10/10/ 2002, 25-30/07/2003 e 29/11-08/12/2003, durante 10, 5 e 12 noites consecutivas, respectivamente, em intervalos de 12 horas seguidas (das 18:00 às 06:00h).

A classificação e a nomenclatura das espécies dos Sphingidae seguem Kitching \& Cadiou (2000). As espécies foram comparadas com figuras de D'Abrera (1986).

O material estudado encontra-se depositado na Coleção de Invertebrados do Instituto Nacional de Pesquisas da Amazônia - INPA. Os indivíduos receberam os seguintes $\mathrm{n}^{\text {os }} \mathrm{de}$ Registro do INPA: 35401-35431; 78501-78764; somando um total de 293 exemplares depositados; os dois indivíduos de Neococytius cluentius (Cramer, 1775) foram doados ao Museu da Ciência de Valência "Príncipe Felipe", Espanha, para a Exposição: Amazônia - o Reino da Diversidade.

\section{RESULTADOS E DISCUSSÃO}

Foram colecionados 295 exemplares de 46 espécies e 20 gêneros, em 27 noites, 324 horas de esforço de coleta (Tabela 1). A tribo Dilophonotini foi a mais representativa com 23 espécies distribuidas em 11 gêneros (89 indivíduos, 30 \%; 66 machos e 23 fêmeas), seguida por Sphingini 9 spp em 4 gêneros ( 57 indivíduos. 19.2\%; $42 \mathrm{M} \mathrm{e} \mathrm{15} \mathrm{F),} \mathrm{Macroglossini} 6$ spp. em 1 gênero (58 indivíduos. $19.7 \% 51 \mathrm{M} \mathrm{e} 7 \mathrm{~F}$ ), Philampelini 4 spp. em 1 gênero (20 indivíduos. 6.9\%; 15M e 5F), Ambulycini 3 spp. em 2 gêneros (69 indivíduos 23.3\%; 57 M e 12 F) e Acherontiini 1 sp. 1 gênero. (2 indivíduos, 2 M) Tabela 2.

Dentre as espécies de mariposas esfingídeas ocorrentes em Beruri, foram registradas pragas (estas assinaladas com *) e as demais, pragas em potencial de plantas de interesse econômico; referindo suas plantas alimento com os nomes comuns (quando têm), nome científico e família botânica (Moss, 1920: Motta, 1993) Tabela 1:

1 - *Agrius cingulata (Fabricius, 1775) - batata-doce Ipomoea batatas (Convolvulaceae); estramônio ou erva-do-diabo Datura sp. (Solanaceae).

2 - Amphimoea walkeri (Boisduval, [1875]) - pião-roxo e pião branco - Jatropha spp. (Euphorbiaceae); anonáceas (Annonaceae).

3-Callionima inuus (Rothschild \& Jordan, 1903) - pepino- 
Tabela 1 - Espécies de Sphingidae registradas em 30/09-10/10/2002 (1 $1^{\mathrm{a}}$ coleta), 25-30/07/2003 (2a coleta) e 29/11-08/12/2003 (3a coleta) no Município de Beruri, Estado do Amazonas, Brasil. ( $\mathrm{M}=$ machos; $\mathrm{F}=$ fêmeas; spp.=espécies $)$

\begin{tabular}{|c|c|c|c|c|c|c|c|c|}
\hline \multirow{2}{*}{$\begin{array}{l}\text { Node } \\
\text { spp. }\end{array}$} & \multirow{2}{*}{ ESPÉCIES } & M & $\mathbf{F}$ & M & $\mathbf{F}$ & M & $\mathbf{F}$ & Total \\
\hline & & \multicolumn{2}{|c|}{$1^{\circ}$ período } & \multicolumn{2}{|c|}{$2^{\circ}$ período } & \multicolumn{3}{|c|}{$3^{\circ}$ período } \\
\hline $1-$ & Adhemarius palmeri (Boisduval, [1875]) & 2 & - & 5 & 1 & 1 & - & 9 \\
\hline $2-$ & Agrius cingulata (Fabricius, 1775)* & - & - & 2 & - & - & - & 2 \\
\hline 3 - & Amphimoea walkeri (Boisduval, [1875]) & - & - & - & 1 & - & - & 1 \\
\hline $4-$ & Callionima inuus (Rothschild \& Jordan, 1903) & 1 & - & - & - & - & - & 1 \\
\hline $5-$ & Callionima nomius (Walker, 1856) & - & - & 1 & - & - & - & 1 \\
\hline $6-$ & Callionima parce (Fabricius, 1775) & 1 & - & 2 & 1 & - & - & 4 \\
\hline $7-$ & Callionima p. pan (Cramer, 1779) & - & - & - & - & - & 1 & 1 \\
\hline $8-$ & Cocytius duponchel (Poey, 1832)* & 1 & - & 15 & 9 & 7 & 1 & 33 \\
\hline $9-$ & Enyo l. lugubris (Linnaeus, 1771) & - & - & - & 2 & - & - & 2 \\
\hline $10-$ & Enyo ocypete (Linnaeus, 1758) & - & - & 2 & 2 & - & - & 4 \\
\hline $11-$ & Erinnyis a. alope (Drury, 1773)* & 1 & - & 6 & 4 & - & - & 11 \\
\hline $12-$ & Erinnyis domingonis (Butler, 1875) & - & - & 1 & - & - & - & 1 \\
\hline $13-$ & Erinnyis e. ello (Linnaeus, 1758)* & 2 & - & 1 & 4 & 2 & - & 9 \\
\hline $14-$ & Erinnyis o. obscura (Fabricius, 1775) & 3 & - & 3 & - & 6 & 2 & 14 \\
\hline $15-$ & Erinnyis oenotrus (Cramer, 1780) & - & - & 2 & - & - & - & 2 \\
\hline $16-$ & Eumorpha anchemolus (Cramer, 1779) & - & - & 3 & 4 & - & - & 7 \\
\hline $17-$ & Eumorpha capronnieri (Boisduval, [1875]) & - & - & 2 & - & - & - & 2 \\
\hline $18-$ & Eumorpha fasciatus (Sulzer, 1776) & - & - & 1 & 1 & - & - & 2 \\
\hline 19 - & Eumorpha v. vitis (Linnaeus, 1758) & - & - & 9 & - & - & - & 9 \\
\hline $20-$ & Isognathus excelsior (Boisduval, 1875) & 3 & - & - & - & - & - & 3 \\
\hline $21-$ & Isognathus leachii (Swainson, 1823)* & 1 & - & - & - & - & - & 1 \\
\hline $22-$ & Madoryx b. bubastus (Cramer, 1777) & - & - & 1 & - & - & - & 1 \\
\hline 23 - & Madoryx plutonius (Hübner, [1819]) & - & - & 1 & - & - & - & 1 \\
\hline $24-$ & Manduca brunalba (Clark, 1929) & - & - & 1 & - & - & - & 1 \\
\hline $25-$ & Manduca diffissa tropicalis (Roth. \& Jordan, 1903) & 1 & - & 4 & - & - & - & 5 \\
\hline $26-$ & Manduca h. hannibal (Cramer, 1779) & - & - & 3 & - & 1 & 1 & 5 \\
\hline $27-$ & Manduca l. lefeburii (Guérin-Ménéville, [1844]) & - & - & 1 & - & - & - & 1 \\
\hline $28-$ & Manduca lucetius (Cramer, 1780) & - & - & 1 & - & - & - & 1 \\
\hline 29 - & Manduca sexta paphus (Cramer, 1779) & - & - & 1 & - & 3 & - & 4 \\
\hline $30-$ & Neococytius cluentius (Cramer, 1775)* & 2 & - & 2 & 3 & - & - & 7 \\
\hline $31-$ & Oryba kadeni (Schaufuss, 1870) & - & - & - & - & - & 1 & 1 \\
\hline $32-$ & Pachylia darceta Druce, 1881 & - & - & 4 & - & 1 & - & 5 \\
\hline $33-$ & Pachylia ficus (Linnaeus, 1758) & - & - & 2 & - & - & - & 2 \\
\hline $34-$ & Pachylioides resumens (Walker, 1856) & - & - & 1 & - & - & - & 1 \\
\hline $35-$ & Perigonia l. lusca (Fabricius, 1777) & - & - & 1 & - & - & - & 1 \\
\hline $36-$ & Perigonia passerina Boisduval, [1819] & - & - & - & - & - & 1 & 1 \\
\hline $37-$ & Phyxus caicus (Cramer, 1777) & 2 & 1 & - & - & 1 & - & 4 \\
\hline $38-$ & Protambulyx eurycles (Herrich-Schäffer, [1854]) & 2 & - & 8 & 1 & - & - & 11 \\
\hline $39-$ & Protambulyx strigilis (Linnaeus, 1771)* & 4 & 1 & 34 & 9 & 1 & - & 49 \\
\hline $40-$ & Pseudosphin tetrio (Linnaeus, 1771)* & - & - & 12 & 5 & - & - & 17 \\
\hline $41-$ & Xylophanes chiron nechus (Cramer, 1777) & 1 & 1 & 22 & 4 & 2 & - & 30 \\
\hline $42-$ & Xylophanes loelia (Druce, 1878) & - & - & 4 & 1 & - & - & 5 \\
\hline
\end{tabular}




\section{ACTA \\ AMAZONICA}

ESFINGÍDEOS (LEPIDOPTERA, SPHINGIDAE) DO

MUNICÍPIO DE BERURI, AMAZONAS, BRASIL

\begin{tabular}{|c|c|c|c|c|c|c|c|c|}
\hline \multirow{2}{*}{$\begin{array}{l}\text { Node } \\
\text { spp. }\end{array}$} & \multirow[t]{2}{*}{ ESPÉCIES } & \multirow{2}{*}{\multicolumn{2}{|c|}{$\begin{array}{l}\text { M F } \\
1^{\circ} \text { período }\end{array}$}} & \multirow{2}{*}{\multicolumn{2}{|c|}{$\frac{M \quad F}{2^{\circ} \text { período }}$}} & \multirow{2}{*}{\multicolumn{3}{|c|}{$\begin{array}{l}M \quad F \\
3^{\circ} \text { período }\end{array}$}} \\
\hline & & & & & & & & \\
\hline $43-$ & Xylophanes obscurus Rothschild \& Jordan, $1910^{* *}$ & - & - & - & 1 & - & - & 1 \\
\hline $44-$ & Xylophanes pistacina (Boisduval, [1875]) & 1 & - & - & - & - & - & 1 \\
\hline $45-$ & Xylophanes t. tersa (Linnaeus, 1771) & - & - & 18 & - & 1 & - & 19 \\
\hline $46-$ & Xylophanes t. thyelia (Linnaeus, 1758) & - & - & 2 & - & - & - & 2 \\
\hline Total & & 28 & 3 & 178 & 53 & 26 & 7 & 295 \\
\hline
\end{tabular}

Tabela 2 - Classificação dos gêneros de Sphingidae. segundo Kitching \& Cadiou (2000), coletados em três períodos $\left(1^{\circ}\right.$ em 2002 e $2^{\circ}$ e $3^{\circ}$ em 2003) no Município de Beruri, Estado do Amazonas, Brasil. M=machos; F=Fêmeas; exs.= e-exemplares; spp=espécies

\begin{tabular}{|c|c|c|c|c|c|c|}
\hline SUBFAMÍLIAS & TRIBOS & SUBTRIBOS & GÊNEROS & $M$ & $\mathbf{F}$ & E-s. \\
\hline SMERINTHINAE & Ambulycini & & Adhemarius & 8 & 1 & 9 \\
\hline 69 exs. & 3 spp. & & Protambulyx & 49 & 11 & 60 \\
\hline SPHINGINAE & Sphingini & & Amphimoea & - & 1 & 1 \\
\hline \multirow[t]{5}{*}{59 exs. } & 9 spp. & & Cocytius & 23 & 10 & 33 \\
\hline & & & Manduca & 15 & 1 & 16 \\
\hline & & & Neococytius & 4 & 3 & 7 \\
\hline & Acheronthiini & & Agrius & 2 & - & 2 \\
\hline & $1 \mathrm{sp}$. & & & & & \\
\hline MACROGLOSSINAE & Dilophonotini & Dilophonotina & Callionima & 5 & 2 & 7 \\
\hline \multirow[t]{14}{*}{167 exs. } & 23 spp. & & Enyo & 2 & 4 & 6 \\
\hline & & & Erinnyis & 27 & 10 & 37 \\
\hline & & & Isognathus & 4 & - & 4 \\
\hline & & & Madoryx & 2 & - & 2 \\
\hline & & & Oryba & - & 1 & 1 \\
\hline & & & Pachylia & 7 & - & 7 \\
\hline & & & Pachylioides & 2 & - & 2 \\
\hline & & & Perigonia & 2 & - & 2 \\
\hline & & & Phryxus & 3 & 1 & 4 \\
\hline & & & Pseudosphinx & 12 & 5 & 17 \\
\hline & Philampelini & & Eumorpha & 15 & 5 & 20 \\
\hline & 4 spp. & & & & & \\
\hline & Macroglossini & Choerocampina & Xylophanes & 51 & 7 & 58 \\
\hline & 6 spp. & & & & & \\
\hline Totais & 45 & & 20 & 233 & 62 & 295 \\
\hline
\end{tabular}

do-mato - Ambelania tenuiflora (Apocynaceae).

4 - *Cocytius duponchel (Poey, 1832) - araticum - Annona montana; graviola - Annona muricata; Annona reticulata; ata, pinha-Annona squamosa; biribá-Rollinia mucosa; Rollinia ortbopetala (Annonaceae).

5-Enyo lugubris lugubris (Linnaeus, 1771) - videira ou parreira - Vitis sp.; Cissus spp.; carão ou cortina-japonesa -
Cissus sicyoides e C. erosus (Vitaceae). Spermacoce sp., jenipapo - Genipa americana (Rubiaceae).

6 -Enyo ocypete (Linnaeus, 1758) - parreira ou videira - Vitis sp.; carão - Cissus spp. (Vitaceae). Davilla spp.;cipó-de-fogo,cipócaboclo ou cipó-de-carijó-Davilla rugosa (Dilleniaceae).

7 - *Erinnyis alope alope (Drury, 1773) - pião-roxo, piãobranco - Jatropha spp.; seringueiras - Hevea spp. e Hevea 


\section{ACTA \\ AMAZONICA}

ESFINGÍDEOS (LEPIDOPTERA, SPHINGIDAE) DO MUNICÍPIO DE BERURI, AMAZONAS, BRASIL brasiliensis; macaxeira ou mandioca ou aipim - Manibot utilissima e Manibot esculenta (Euphorbiaceae). Mamoeiro (Caricaceae). Dedal-de-dama - Allamanda sp. (Apocynaceae). Algodoeiro-Gossypium herbaceum (Malvaceae).

8 - *Erinnyis ello ello (Linnaeus, 1758) - pião branco e roxo -Jatropha spp.; seringueira - Hevea sp. e Hevea brasiliensis; avelós-Euphorbiagymnoclada; macaxeira-Manibot utilissima, Manibot esculenta, Manibot aipi, Manibot flabellifolia, maniçoba - Manibot glaziovii; Euphorbia spp.; carrapateira, palma-de-cristo ou mamona - Ricinus communis; faveleira Cnidosculus fragrans; Hippomane mancinella; nogueira-daíndia, nogueira-brasileira - Aleurites spp.; Sapium sp. (Euphorbiaceae). Mamoeiro - Carica papaya (Caricaceae). Tomateiro - Lycopersicum esculentum, Solanum sp., petunia Petunia sp., fumo ou tabaco-Nicotiana tabacum (Solanaceae). Abiu - Lucuma caimito (Sapotaceae). Dedal-de-dama Allamanda spp., Thevetia sp. (Apocynaceae). Cebola - Allium cepa, pita - Yucca sp. (Liliaceae). Algodoeiro - Gossypium berbaceum (Malvaceae). Figueiras - Ficus sp. (Moraceae). Goiabeiras - Psidium spp. (Myrtaceae).

9-Erinnyis obscura obscura (Fabricius, 1775) - Gonolobus sp.; paina-de-seda - Araujia sericifera; Pbilibertia spp.; Cyancbum palustre; Sarcostemma angustissima (Asclepiadaceae).

10 - Erinnyis oenotrus (Cramer, 1780) - pau-de-colher Zschokkea sp.; Echites sp.; espirradeira ou oleandro-Nerium oleander (Apocynaceae). Morrenia sp.; paina-de-seda-Araujia sericifera; falsa-erva-de-rato ou oficial-de-sala - Asclepias curassavica; cipó-de-leite - Oxypetalum sp. (Asclepiadaceae).

11 - Eumorpha anchemolus (Cramer, 1779) - carão - Cissus spp.; videira-Vitis sp. (Vitaceae).

12 - Eumorpha fasciatus (Sulzer, 1776) - cruz-de-malta Jussieua linefolia,Jussieua latifolia (Onagraceae).

13 - Eumorpha vitis vitis (Linnaeus, 1758) - cortina-japonesa ou carão - Cissus sycioides; videira ou parreira - Vitis sp. (Vitaceae).

14 - Isognathus excelsior (Booisduval, 1875) - sucuba ou janaguba - Himatantbus sucuuba (Apocynaceae).

15 - *Isognatbus leachii (Swainson, 1823) - dedal-de-dama - Allamanda catbartica; dedal-de-jacobina - Allamanda blanchetii; 4 patacas roxa-Allamanda violacea; sucuba ou janaguba - Himatantbus sucuuba (Apocynaceae).

16-Madoryx plutonius (Hübner, [1819]) - canela-de-velho - Miconia minutiflora (Melastomataceae).

17-Manduca brunalba (Clark, 1929) - pau-de-formiga ou grão-de-galo - Cordia sp. (Boraginaceae). Rollinia ortbopetala (Annonaceae).

18-Manduca diffissa tropicalis (Rothschild \& Jordan, 1903) - jurubebas - Solanum camponiforme (Solanaceae).

19 - Manduca hannibal hannibal (Cramer, 1779) solanáceas (jurubebas e outras) Solanum campaniforme, Cestrum floribundum, erva-do-inferno - Datura cornigera (Solanaceae). Clerodendron fragans e Aegiphila cuspidata (Verbenaceae).
20 - Manduca lucetius (Cramer, 1780) - solanáceas Solanum spp. (Solanaceae).

21 - *Manduca sexta papbus (Cramer, 1779) - muitas espécies de solanáceas: pimenteira, pimentão-Capsicum spp., tomateiro - Lycopersicum esculentum, fumo ou tabaco Nicotiana tabacum (Solanaceae).

22 - *Neococytius cluentius (Cramer, 1775) - Graviola Annona muricata; Annona reticulata; cipoíra - Guatteria sp (Annonaceae). Pimenta-longa - Piper aduncum-Piperaceae.

23 - Oryba kadeni (Schaufuss, 1870) - Palicourea sp., Remija sp. (Rubiaceae). Coccoloba latifolia (Polygonaceae).

24 - Pachylia ficus (Linnaeus, 1758) - figueiras - Ficus spp., Ficus benjamina; jaqueira - Artocarpus integrifolia (Moraceae).

25-Pachylioides resumens (Walker, 1856) - pau-de-colher -Zschokkea sp., Echites sp. - Apocynaceae. Figueiras -Ficus spp. (Moraceae).

26-Perigonia lusca lusca (Fabricius, 1777) - unha-de-gavião - Ouroparia guianensis e café Coffea arabica, Coffea liberica (Rubiaceae).

27 - Phyxus caicus (Cramer, 1777) - pau-de-colher Zschokkea sp., Echites sp. (Apocynaceae).

28-Protambulyx eurycles (Herrich-Schäffer, [1854]) -breu - Tapirira sp. (Anacardiaceae).

29 - *Protambulyx strigilis (Linnaeus, 1771) - taperebá Spondias lutea, cajarana-Spondias dulcis, cajueiro-Anacardium occidentale (Anacardiaceae). Mamoeiro - Carica papaya (Caricaceae). Guandeiro - Canajus indicus (Fabaceae). Sabugueiro-Sambucus australis (Caprifoliaceae).Erytbroxylon sp. (Erythroxylaceae).

30 - *Pseudosphinx tetrio (Linnaeus, 1771) - jasmim-manga - Plumeria spp.; sucuba, janaguba - Himatantbus sucuuba; jasmins -Jasminum spp.; espirradeira - Nerium oleander (Apocynaceae).

31-Xylophanes chiron nechus (Cramer, 1777) - mamoeiro - Carica papaya (Caricaceae).

32-Xylophanes tersa tersa (Linnaeus, 1771) -Spermacoce glabra; Manettia sp.; Pentas sp. (Rubiaceae).

\section{CONCLUSÕES}

Nas três excursões, das 46 espécies coletadas, foram obtidas 8 espécies que, somados os exemplares (184) representam $62,4 \%$ dos esfingídeos coletados (295 indivíduos): *Protambulyx strigilis (49 exs.); *Cocytius duponchel (33); Xylophanes chiron nechus (30); Xylophanes tersa (19); *Pseudosphinx tetrio (17); Erinnyis obscura (14); *Erinnyis alope (11); Protambulyx eurycles (11). As quatro espécies assinaladas com asterisco são pragas de interesse econômico.

Todas as espécies estão sendo registradas pela primeira vez para o Município de Beruri, Estado do Amazonas. Os números de espécies capturadas e de registros novos em relação ao número de noites de coleta, mostram o quão importante 
são as coletas sistemáticas dessas mariposas. O primeiro exemplar de Xylophanes obscurus Rothschild \& Jordan, $1910 \mathrm{e}$ o quinto de Xylophanes pistacina (Cramer, 1777) foram depositados na coleção do INPA. X. obscurus é pouco coletada à luz e as fêmeas são mais conhecidas que os machos (Kitching \& Cadiou, 2000). Xilophanes pistacina só havia sido coletada na ilha de Maracá, Estado de Roraima (Motta et al., 1991); ainda, segundo Haxaire (1985) esta espécie além de ser pouco comum, só é coletada em áreas com floresta primária.

\section{AGRADECIMENTOS}

Ao Sr. Daniel Garibotti pelo apoio. Aos Srs. Pedro Marinho Neves - Presidente da Associação dos Agropecuários pelos contatos e José Maria Rodrigues Queiroz - Secretário de Produção Rural, proprietário do sítio, na Estrada do INCRA, onde foram realizadas as coletas. Ao técnico do INPA Ulysses Carvalho Barbosa pela amizade e companheirismo.

Àmestranda Gilcélia Melo Lourido pelo auxílio com o mapa.

\section{BIBLIOGRAFIA CITADA}

D'Abrera, B. 1986. Sphingidae Mundi. Hawk Moths of the World. Ed. Classey E. W. Ltd., Oxon, 266 pp.

Haxaire, J. 1985. Le genre Xylophanes Hübner [1819] en Guyane Française (Lepidoptera, Sphingidae). Bull. Soc. Sci. Nat. (Compiègne), 47:21-24, 1 pl.
IBGE 2000. http://www.ibge.gov.br. Consultado em 07/03/2003.

Kitching, I.J.; Cadiou, J.M. 2000. Hawkmoths of the world: an annotated and illustrated revisionary checklist (Lepidoptera: Sphingidae). Cornell University Press, Ithaca, 227 pp.

Motta, C.S. 1993. Mariposas esfingídeas (Lepidoptera, Sphingidae) da Amazônia Brasileira e o Meio Ambiente. In Ferreira, E.J.G., Santos, G.M., Leão, E.L.M. \& Oliveira. L.A. (Eds.). Bases Científicas para Estratégias de Preservação e Desenvolvimento da Amazônia - Fatos e Perspectivas. Vol. 2. Instituto Nacional de Pesquisas da Amazônia, Manaus. p. 141-146.

Motta, C.S.; Ferreira, R.L.M.; Aguiar, N.O. 1991 (1992). Sobre a esfingofauna da ilha de Maracá e da serra de Pacaraima, Roraima (Lepidoptera, Sphingidae). Acta Amazonica, 21: 319-324.

Moss, A.M. 1920. Sphingid of Para. Brasil. Early stages, food plants, habits, etc. Novitates Zoologicae, 27: 333-415.

\author{
RECEBIDO EM 14/06/2005 \\ ACEITO EM 17/11/2005
}

